\title{
A simple and inexpensive pinch meter to detect subclinical weakness among leprosy patients
}

\author{
D. SOARES \& A. RIEDEL \\ Anandaban Leprosy Hospital, The Leprosy Mission, PO Box 151, \\ Kathmandu, Nepal
}

Accepted for publication 3 July 1996

\begin{abstract}
Summary This paper describes the use of a neonatal sphygmomanometer cuff as a simple, inexpensive pinch meter. Normal values for key pinch, pulp pinch and side pinch in the dominant hand of healthy Nepali people are provided.

The pinch meter was also used to test pinch strength in hands affected by leprosy and normal hands. Some patients with leprosy who have no objective weakness on voluntary muscle testing (VMT) have less pinch strength than people without leprosy. The pinch meter is a useful tool for the early detection of motor function loss.
\end{abstract}

\section{Introduction}

Part of the routine assessment of patients with leprosy is an assessment of muscle power in the hand. There are many methods of testing hand function. Voluntary muscle testing (VMT) as described by the Medical Research Council ${ }^{1}$ is a detailed and accurate method of assessing hand muscle function.

However even amongst well-trained observers there is significant interobserver variation. ${ }^{2}$ Another problem in assessment is that the scale $0-5$ is not, in practice, linear, i.e. there is a much greater loss of strength between grade 5-3 than between 31. Staff usually modify the VMT scale for testing the intrinsic muscles of the hand as they are not significantly affected by gravity. Thus Grade 3 = full range of movement, Grade 2 = partial range of movement, and Grade $1=$ muscle flicker but no movement.

Finger dynamometry is an accurate, reproducible method of testing strength in key and pulp pinches. However pinch meters are expensive and way beyond the budgets of most third world hospital.

At Anandaban Leprosy Hospital, Nepal we designed our own pinch meter using a disposable neonatal sphygmomanometer cuff which we received in a box of donated medical supplies. We tested it on normal, healthy hands of people who presented to our nonleprosy outpatients department as well as on hands of people affected by leprosy. 


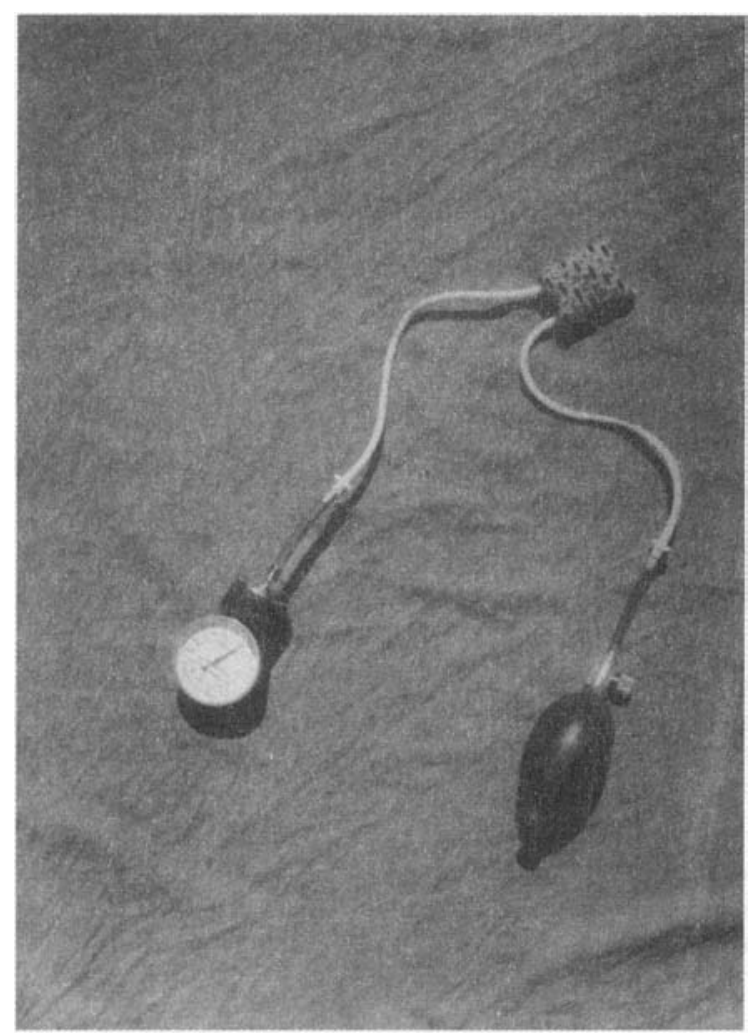

Figure 1. Pinch meter is cloth bag with blood pressure cuff and gauge attached.

\section{Materials}

The sphygmomanometer cuff is a DISPOSA-CUF 3 TM neonatal size 3 (Figure 1). This was fitted inside a cloth bag $(40 \mathrm{~mm}$ by $40 \mathrm{~mm})$ so that the volume of the cuff remained relatively constant. The cuff was connected to an ordinary anaeroid sphygmomanometer. The meter was set at zero and the cuff was then inflated to $20 \mathrm{mmHg}$.

Pulp-to-pulp pinch (between the thumb and each of the fingers) (Figure 2) and key pinch (between the thumb and the side of the index) was measured. Side pinch (Figure 3) between the index and long fingers, long and ring fingers and ring and little fingers was also measured.

The people tested were classified into 4 groups:

Group 1 normal hands in people not affected by leprosy $(n=24)$.

Group 2 affected hands in people affected by leprosy $(n=22)$.

Group 3 normal hands in people affected by leprosy where the other hand was affected by leprosy $(n=17)$.

Group 4 normal hands in people affected by leprosy where the other hand was not affected by leprosy $(n=15)$. 


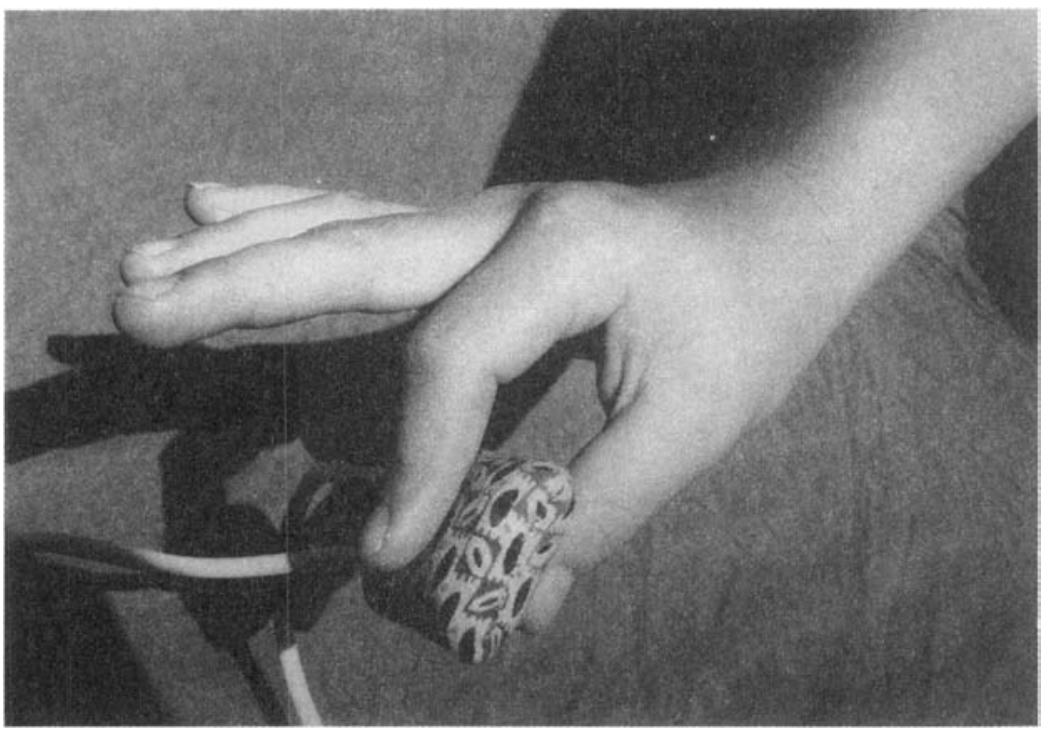

Figure 2. Pulp pinch.

\section{Results}

All measurements listed were made with the cloth bag cover. All strengths were measured in $\mathrm{mmHg}$. These measurements were carried out on 78 hands. People who were tested were aged between 15 and 55 years old.

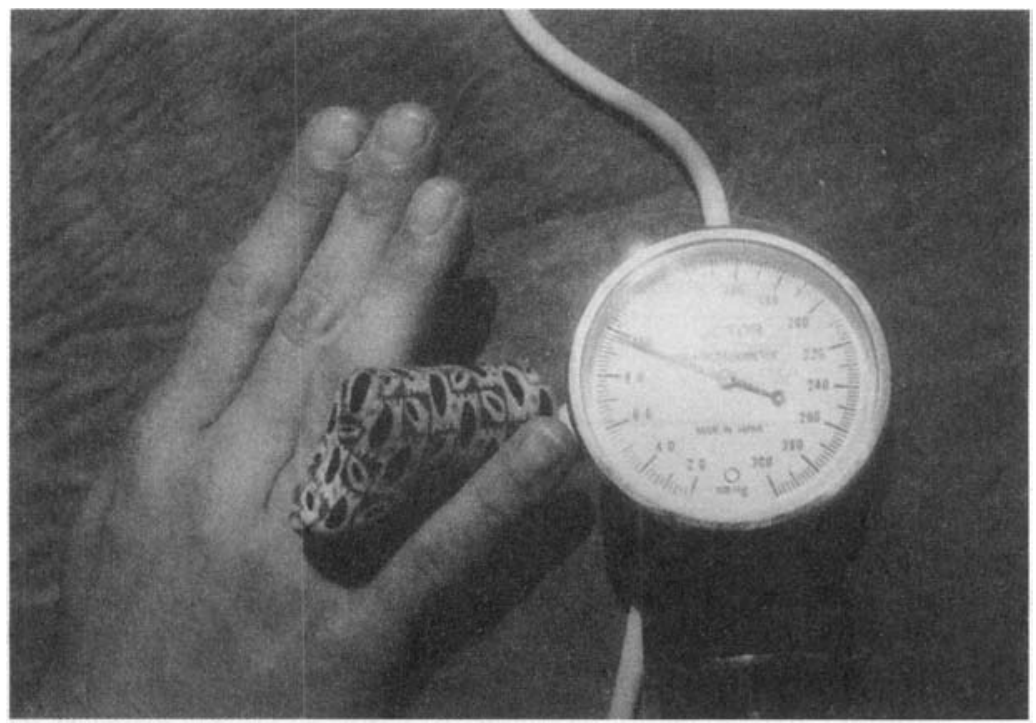

Figure 3. Side pinch. 
Table 1. All measurements are in $\mathrm{mmHg}$ and carried out with the cloth bag cover.

\begin{tabular}{lrrr}
\hline Pulp pinch & Mean & sd & Range \\
\hline Thumb-Index & 237 & 46 & $160-360$ \\
Thumb-Middle & 195 & 45 & $130-320$ \\
Thumb-Ring & 152 & 54 & $70-280$ \\
Thumb-Little & 126 & 31 & $70-180$ \\
Key pinch & 246 & 41 & $170-320$ \\
Side pinch & & & \\
Index-Middle & 126 & 24 & $85-170$ \\
Middle-Ring & 87 & 15 & $60-110$ \\
Ring-Little & 71 & 13 & $40-95$ \\
\hline
\end{tabular}

$\mathrm{SD}$, standard deviation

Table 2. Mean pressure in $\mathrm{mmHg}(\%$ of normal-Group 1$)$

\begin{tabular}{lrrrr}
\hline & Group 1 & Group 2 & Group 3 & Group 4 \\
\hline Pulp pinch & & & & \\
Index & 237 & $130(55 \%)$ & $191(81 \%)$ & $221(93 \%)$ \\
Middle & 195 & $133(68 \%)$ & $194(99 \%)$ & $199(102 \%)$ \\
Ring & 152 & $98(64 \%)$ & $148(97 \%)$ & $148(97 \%)$ \\
Little & 126 & $64(51 \%)$ & $112(89 \%)$ & $121(96 \%)$ \\
Key pinch & 246 & $149(61 \%)$ & $209(85 \%)$ & $251(102 \%)$ \\
Side pinch & & & & \\
Index-Mid. & 126 & $69(55 \%)$ & $102(81 \%)$ & $121(96 \%)$ \\
Middle-Ring & 87 & $52(60 \%)$ & $73(84 \%)$ & $87(100 \%)$ \\
Ring-Little & 71 & $36(51 \%)$ & $59(83 \%)$ & $71(100 \%)$ \\
Grip & 234 & $143(61 \%)$ & $187(80 \%)$ & $193(82 \%)$ \\
& & & & \\
\hline
\end{tabular}

Table 3. Correlation between side pinch between ring and little finger VMT of ring and little finger

\begin{tabular}{lcr}
\hline & \multicolumn{2}{c}{ Side pinch } \\
\cline { 2 - 3 } Finger intrinsics & Normal & Weak \\
\hline Normal & 23 & 6 \\
Weak & 2 & 15 \\
\hline
\end{tabular}

The mean, standard deviation and range of measurements in healthy people (Group 1) are listed in Table 1.

Among hands of people in Group 2 (leprosy affected hands) there were 15 hands with combined lesions (ulnar and median nerve), 6 hands with only ulnar nerve lesions and one hand with only median nerve damage.

A detailed summary of the results is listed in Table 2. Hands affected by leprosy (group 2) have consistently lower strengths as measured by pressure generated. The 
mean pressure in hands affected by leprosy varied from $51 \%$ of that produced by normal hands for Thumb-Little pulp pinch to $68 \%$ of that produced by normal hands for Thumb-Middle pulp pinch. Normal hands in people where the other hand was affected by leprosy (group 3) were also weaker when compared with normal hands in people not affected by leprosy. The mean pressure produced varied from $81 \%$ of that produced by normal hands for Index-Middle side pinch to the same pressure produced by normal hands for Thumb-Middle pulp pinch. In this group the patient pressures could be divided into 2 subgroups - 6 hands with pressure equal to normal hands and 11 hands with pressures below that of normal hands. It is possible that these 11 hands represent hands with early motor weakness.

People affected by leprosy but where both hands were normal had similar means to people not affected by leprosy, except for grip strength ( $82 \%$ of normal grip strength).

There was good correlation between VMT and pinch strength in the side pinches. For example if we code the intrinsic function (lumbrical action) of the little and ring fingers together (total VMT $=10=$ normal, and if less than $10=$ weak) and code the side pinch between these fingers (more than $40 \mathrm{mmHg}=$ normal and less than $40 \mathrm{mmHg}=$ weak) then we arrive at the result given in Table 3. From this there are 6 patients with a normal VMT but with 'weak' side pinch who may represent subclinical weakness.

There was good correlation between ADM weakness and side pinches. Index-Long $r=0.74$; Long-Ring $r=0.66$, Ring-Little $r=0.73$. There was poorer correlation between weakness of OPP and the pulp pinches. A weakness of FDP or of FDS had poor correlation with all of the pinches.

\section{Discussion}

Finger dynamometry is an essential part of the assessment of patients with nerve damage leading to muscle weakness in the hand. The technique of measuring the pinch pressure is simple, reproducible and quick. However as with most medical equipment pinch meters are very expensive. (Prices I have had quoted range from US\$ 500 to 1000, which is beyond the budgets of most hospitals in developing countries). In contrast the disposable neonatal cuffs cost less than $\$ 5$ each. So far the first cuff has lasted more than 6 months with no signs of fatigue.

Occasionally, when encased in the cloth bag, the cuff can be pinched by very strong hands beyond the range of the anaeroid sphygmomanometer. To overcome this problem we have tried using a firm plastic cover made from a film container. This requires greater force to compress it and can thus be used for very strong pinches (in particular the key pinch and the Thumb-Index pulp pinch). The soft cloth bag is more sensitive and suited to side pinches. The use of the neonatal sphygmomanometer cuff makes the use of finger dynamometry available to hand therapists and surgeons in developing countries with limited resources.

In the normal hands of people where the other hand is affected by leprosy the mean strength was lower than in normal hands in normal people. This may be due to early motor nerve damage where the VMT is normal but where there is a measurable deficit in pinch strength. These patients are at risk of developing overt motor weakness if they experience further damage due to leprosy neuritis. In these patients finger dynamometry 
may be a useful tool to assess early changes in motor strength. This technique may also be useful for assessment of recovery of nerve function after prednisolone or peripheral nerve surgery because it is less liable to assessor bias.

\section{Conclusion}

Finger dynamometry using a neonatal sphygmomanometer cuff is reliable and useful in the assessment of hand function, particularly in situations where resources are limited.

This simple, inexpensive pinch meter is a useful addition to the tools available for the assessment of hand motor function in people affected by leprosy. It may help to identify those at risk of further nerve damage who may later develop overt motor weakness as measured by VMT.

\section{References}

${ }^{1}$ Medical Research Council. Aids to the examination of the peripheral nervous system. Memorandum No. 45. Her Majesty's Stationery Office. 1975.

${ }^{2}$ Leinhardt C, Currie H, Pasquier R, Butlin R, Wheeler J. Inter-observer variability in the assessment of nerve function in leprosy patients in Ethiopia and Nepal. Int J Lepr, 61; 145. Paper presented at 14th ILA congress, Florida.

${ }^{3}$ Critikon Dispos-A-CuF: Johnson and Johnson Medical Ltd, Coronation Road, Ascot, Berks SL5 9EY, UK. 